\title{
Restriction of Toeplitz Operators on Their Reducing Subspaces
}

\author{
Anjian $\mathrm{Xu}^{1}$ and Yang $\mathrm{Zou}^{2}$ \\ ${ }^{1}$ College of Science, Chongqing University of Technology, Chongqing 400065, China \\ ${ }^{2}$ School of Mathematics and Information, Chongqing University of Education, Chongqing 400054, China \\ Correspondence should be addressed to Anjian Xu; xuaj@cqut.edu.cn
}

Received 26 May 2017; Accepted 6 August 2017; Published 7 September 2017

Academic Editor: Raúl E. Curto

Copyright (C) 2017 Anjian Xu and Yang Zou. This is an open access article distributed under the Creative Commons Attribution License, which permits unrestricted use, distribution, and reproduction in any medium, provided the original work is properly cited.

We study the restrictions of analytic Toeplitz operator on its minimal reducing subspaces for the unit disc and construct their models on slit domains. Furthermore, it is shown that $T_{z^{n}}$ is similar to the sum of $n$ copies of the Bergman shift.

\section{Introduction}

Let $T$ be a bounded linear operator on a Hilbert space $\mathscr{H}$; a subspace $\mathfrak{M}$ of $\mathscr{H}$ is called an invariant subspace of $T$ if $T(\mathfrak{M}) \subseteq \mathfrak{M}$ and a reducing subspace of $T$ if $\mathfrak{M}$ is an invariant subspace of $T$ and $T^{*}$. A reducing subspace $\mathfrak{M}$ of $T$ is called minimal if for every reducing subspace $\widetilde{\mathfrak{M}}$ of $T$ such that $\widetilde{\mathfrak{M}} \subseteq$ $\mathfrak{M}$ then either $\widetilde{\mathfrak{M}}=\mathfrak{M}$ or $\widetilde{\mathfrak{M}}=0$. For a concrete bounded operator $T$ on a separable Hilbert space $\mathscr{H}$, it is important to determine invariant subspaces and reducing subspaces for $T$.

Let $\mathbb{D}$ be the unit open disc in the complex plane and $d A(z)$ be the normalized area measure on $\mathbb{D}$. The Bergman space $L_{a}^{2}(\mathbb{D})$ consists of all analytic functions in the Lebesgue space $L^{2}(d A(z))$. It is clear that $L_{a}^{2}(\mathbb{D})$ is a closed subspace of $L^{2}(d A(z))$, and let $P$ denote the projection from $L^{2}(d A(z))$ onto $L_{a}^{2}(\mathbb{D})$. The Toeplitz operator $T_{\varphi}$ on $L_{a}^{2}(\mathbb{D})$ with symbol $\varphi \in L^{\infty}(d A(z))$ is defined by $\left(T_{\varphi} f\right)(z)=P(\varphi f)(z)$; it is called an analytic Toeplitz operator if $\varphi \in H^{\infty}(\mathbb{D})$.

An $n$ th-order Blaschke product $B$ is the analytic function on $\mathbb{D}$ given by

$$
B(z)=e^{i \theta} \prod_{i=1}^{n} \frac{z-a_{i}}{1-\bar{a}_{i} z},
$$

where $\theta$ is a real number and $a_{i} \in \mathbb{D}$ for $1 \leq i \leq n$. A Blaschke product is very important in the theory of Hardy space.

Characterization of reducing subspaces of an analytic Toeplitz operator $T_{\varphi}$ on Bergman space has been of great interest for last two decades. Thomson $[1,2]$ showed that it suffices to study reducing subspace of $T_{B}$ for a finite Blaschke product in the case of Hardy space. It can be generalized to Bergman spaces easily.

Zhu studied the reducing subspaces of $T_{B}$ for a Blaschke product of order 2 firstly and showed that $T_{B}$ has exactly two distinct minimal reducing subspaces (cf. [3]). Motivated by this fact, Zhu conjectured that the number of minimal reducing subspaces of $T_{B}$ equals the order of $B$ (cf. [3]). Guo et al. showed that in general this is not true (cf. [4]), and they found that the number of minimal reducing subspaces of $T_{B}$ equals the number of connected components of the Riemann surface of $B(z)=B(w)$ when the order of $B$ is $3,4,6$. Then they conjectured that the number of minimal reducing subspaces of $T_{B}$ equals the number of connected components of the Riemann surface of $B(z)=B(w)$ for any finite Blaschke product (called the refined Zhu's conjecture, cf. [4]). Douglas et al. confirmed the conjecture in $[5,6]$ by using local inverses of Blaschke products [7]. Tikaradze [8] generalized a part of results in [5] to bounded smooth pseudoconvex domains in $\mathbb{C}^{N}$. Douglas and Kim [9] studied reducing subspace $T_{z^{n}}$ on Bergman space of the annulus; the case of Hardy space was summarized in [10].

In [11], Douglas et al. generalized the bundle shift [12] to the case of Bergman spaces, constructed a vector bundle model for analytic Toeplitz operator $T_{\varphi}$ on the Bergman space $L_{a}^{2}(\mathbb{D})$, and tried to build vector bundle models for restrictions of $T_{\varphi}$ to its minimal reducing subspaces, but it is not completed. Douglas [13] studied unitary equivalence of the restrictions by computing their curvatures of corresponding 
geometric models. Hu et al. [14] showed that, for $T_{\varphi}$, there is a distinguished reducing subspace $\mathscr{M}_{0}$ such that the restriction of $T_{\varphi}$ on $\mathscr{M}_{0}$ is the Bergman shift. In this paper, we analyze the concrete examples to see what are the possible models for these restrictions for further research.

\section{Unitary Equivalence and Similarity of Weighted Shifts}

Let $S$ be an operator on a separable infinite-dimensional Hilbert space $\mathscr{H} . S$ is called a one-side weighted shift if there exist an orthonormal basis $\left\{e_{i}\right\}, i=0,1,2, \ldots$ for $\mathscr{H}$ and a bounded sequence $\left\{\alpha_{i}\right\}$ of complex numbers such that $S e_{i}=$ $\alpha_{i} e_{i+1}$. Similarly, $S$ is called a two-side weighted shift if there exist an orthonormal basis $\left\{e_{i}\right\}, i=\ldots,-2,-1,0,1,2, \ldots$ for $\mathscr{H}$ and a bounded sequence $\left\{\alpha_{i}\right\}$ of complex numbers such that $S e_{i}=\alpha_{i} e_{i+1}$ for all $i \in \mathbb{Z}$.

Lemma 1 (see [15]). Suppose that $S$ and $T$ are two injective one-side weighted shifts with weights $\left\{\alpha_{i}\right\}$ and $\left\{\beta_{i}\right\}$ respectively; then $S$ is unitarily equivalent to $T$ if and only if $\left|\alpha_{i}\right|=\left|\beta_{i}\right|$ for all $i$.

Lemma 2 (see [15]). Suppose that $S$ and $T$ are two injective one-side weighted shifts with weights $\left\{\alpha_{i}\right\}$ and $\left\{\beta_{i}\right\}$, respectively; then $S$ is similar to $T$ if and only if there exist two constants $M$ and $N$ such that

$$
0<M \leq \prod_{j=0}^{i}\left|\frac{\beta_{j}}{\alpha_{j}}\right| \leq N<\infty
$$

for all $i$.

\section{Models for Restriction of Toeplitz Operators on Their Minimal Reducing Subspaces}

3.1. The Bergman Spaces on the Slit Disc. The domain $G=$ $\mathbb{D} \backslash[0,1)$ is called the slit disk. Let $d A$ denote the normalized area measure on $G$. $L_{a}^{2}(G)$ is the set of analytic functions in the Lebesgue space $L^{2}(G, d A)$. For a nonnegative measurable function $g(w)$ on $G$, we can define the weighted Bergman space with respect to $g(w) d A(w)$ to be the set of all analytic functions in the Lebesgue space $L^{2}(G, g(w) d A)$. Ross studied invariant subspaces of Bergman spaces on slit domains in [16]. Aleman et al. defined and studied the Hardy space of a slit domain and in particular they studied the invariant subspace of the slit disk; one can consult [17] for details.

3.2. Slit Disc Models for $M_{z^{2}}$. It is easy to check that $1 / 4|w|$ is a measurable function on $G$ and that $(1 / 4|w|) d A(w)$ is a probability measure on $G$.

Lemma 3. The Bergman space $L_{a}^{2}(G,(1 / 4|w|) d A(w))$ contains constants and power functions $\left\{w^{n} \mid n \in \mathbb{N}\right\}$. Furthermore, $\left\{\sqrt{2(2 n+1)} w^{n} ; n \in \mathbb{N}\right\}$ and $\left\{2 \sqrt{n+1} w^{n} ; n \in\right.$ $\mathbb{N}\}$ are orthonormal basis of $L_{a}^{2}(G,(1 / 4) d A(w))$ and $L_{a}^{2}(G,(1 / 4|w|) d A(w))$, respectively.
Proof. For the Bergman space $L_{a}^{2}(G,(1 / 4|w|) d A(w))$ and $n \epsilon$ $\mathbb{N}$,

$$
\begin{aligned}
\left\|w^{n}\right\|^{2} & =\int_{\mathbb{G}}\left|w^{n}\right|^{2} \frac{1}{4|w|} d A(w)=\frac{1}{4 \pi} \int_{0}^{1} r^{2 n} d r \int_{0}^{2 \pi} d \theta \\
& =\frac{1}{2(2 n+1)},
\end{aligned}
$$

so $\left\|w^{n}\right\|=1 / \sqrt{2(2 n+1)}$.

For the Bergman space $L_{a}^{2}(G,(1 / 4) d A(w))$ and $n \in \mathbb{N}$,

$$
\begin{aligned}
\left\|w^{n}\right\|^{2} & =\int_{\mathbb{G}}\left|w^{n}\right|^{2} \frac{1}{4} d A(w)=\frac{1}{4 \pi} \int_{0}^{1} r^{2 n+1} d r \int_{0}^{2 \pi} d \theta \\
& =\frac{1}{4(n+1)},
\end{aligned}
$$

so $\left\|w^{n}\right\|=1 / 2 \sqrt{(n+1)}$.

Lemma 4. The multiplication operator $T_{w}$ is a bounded operator on $L_{a}^{2}(G,(1 / 4|w|) d A(w))$ and $L_{a}^{2}(G,(1 / 4) d A(w))$.

Proof. For the Bergman space $L_{a}^{2}(G,(1 / 4|w|) d A(w))$, it is easy to show that

$$
\begin{aligned}
T_{w}\left(\sqrt{2(2 n+1)} w^{n}\right) & =\sqrt{2(2 n+1)} w^{n+1} \\
& =\sqrt{\frac{2 n+1}{2 n+3}}\left(\sqrt{2(2 n+3)} w^{n+1}\right) .
\end{aligned}
$$

So $T_{w}$ is a weighted shift with weight $\{\sqrt{(2 n+1) /(2 n+3)}\}_{n=0}^{\infty}$ and $\left\|T_{w}\right\| \leq 1$. Similarly, on the Bergman space $L_{a}^{2}(G,(1 / 4) d A(w)), T_{w}$ is a weighted shift with weight $\{\sqrt{(n+1) /(n+2)}\}_{n=0}^{\infty}$ and so bounded.

We know that

$$
\begin{aligned}
& \mathscr{M}_{0}=\bigvee\left\{z^{k} ; k \equiv 0 \bmod 2\right\}, \\
& \mathscr{M}_{1}=\bigvee\left\{z^{k} ; k \equiv 1 \bmod 2\right\}
\end{aligned}
$$

are the only two minimal reducing subspaces of $T_{z^{2}}$ on $L_{a}^{2}(\mathbb{D})$.

Theorem 5. $\left.T_{z^{2}}\right|_{\mathscr{M}_{0}},\left.T_{z^{2}}\right|_{\mathscr{M}_{1}}$ are unitarily equivalent to the multiplication operators $T_{w}$ on the following two spaces, respectively:

$$
\begin{gathered}
L_{a}^{2}\left(G, \frac{1}{4|w|} d A(w)\right), \\
L_{a}^{2}\left(G, \frac{1}{4} d A(w)\right) .
\end{gathered}
$$

Proof. Define the following maps:

$$
\begin{aligned}
L_{a}^{2}\left(G, \frac{1}{4} d A(w)\right) & \longrightarrow \mathscr{M}_{1} \\
V_{1}: f(w) & \longmapsto z f\left(z^{2}\right) \\
L_{a}^{2}\left(G, \frac{1}{4|w|} d A(w)\right) & \longrightarrow \mathscr{M}_{0} \\
V_{0}: f(w) & \longmapsto f\left(z^{2}\right) .
\end{aligned}
$$


Then $V_{1}, V_{0}$ are two isometries on $\mathscr{M}_{1}, \mathscr{M}_{0}$, respectively. As a fact, by changing of variable $z^{2}=w,|d z / d w|=|1 / 2 \sqrt{w}|$, we have

$$
\begin{aligned}
\left\|V_{1} f(w)\right\|^{2} & =\left\|z f\left(z^{2}\right)\right\|^{2}=\int_{\mathbb{D}}\left|z f\left(z^{2}\right)\right|^{2} d A(z) \\
& =\int_{G}|\sqrt{w} f(w)|^{2}\left|\frac{d z}{d w}\right|^{2} d A(w) \\
& =\int_{G}|\sqrt{w} f(w)|^{2}\left|\frac{1}{2 \sqrt{w}}\right|^{2} d A(w) \\
& =\int_{G}|f(w)|^{2} \frac{1}{4} d A(w)=\|f(w)\|^{2},
\end{aligned}
$$

so $V_{1}$ is an isometry. And

$$
\begin{aligned}
\left\|V_{0} f(w)\right\|^{2} & =\left\|f\left(z^{2}\right)\right\|^{2}=\int_{\mathbb{D}}\left|f\left(z^{2}\right)\right|^{2} d A(z) \\
& =\int_{G}|f(w)|^{2}\left|\frac{d z}{d w}\right|^{2} d A(w) \\
& =\int_{G}|f(w)|^{2}\left|\frac{1}{2 \sqrt{w}}\right|^{2} d A(w) \\
& =\int_{G}|f(w)|^{2} \frac{1}{4|w|} d A(w)=\|f(w)\|^{2} ;
\end{aligned}
$$

it shows that $V_{0}$ is an isometry. The next is to show that $T_{z^{2}} V_{1}=V_{1} T_{w}$ on $L_{a}^{2}(G,(1 / 4) d A(w))$. For every function $f(w) \in L_{a}^{2}(G,(1 / 4) d A(w))$, we have

$$
\begin{aligned}
T_{z^{2}} V_{1} f(w) & =T_{z^{2}} z f\left(z^{2}\right)=z^{3} f\left(z^{2}\right)=z\left(z^{2} f\left(z^{2}\right)\right) \\
& =V_{1}(w f(w))=V_{1} T_{w} f(w)
\end{aligned}
$$

One can show that $T_{z^{2}} V_{0}=V_{0} T_{w}$ on $L_{a}^{2}(G,(1 / 4 w) d A(w))$ similarly.

Suppose that $\varphi$ is a Blaschke product $\varphi=((a-z) /(1-$ $\bar{a} z))((b-z) /(1-\bar{b} z))=\varphi_{a}(z) \varphi_{b}(z)$ with two different zeroes $a$ and $b$; let $k_{\lambda}(z)=\left(1-|\lambda|^{2}\right) /(1-\bar{\lambda} z)^{2}$ be the normalized reproducing kernel at $\lambda$.

Lemma 6 (see [3]). Let $\varphi=((a-z) /(1-\bar{a} z))((b-z) /(1-$ $\bar{b} z))$ with two different zeroes $a, b \in \mathbb{D}$. Let $m$ be the geodesic midpoint between $a$ and $b$. Then the operator

$$
M_{\varphi}: L_{a}^{2}(\mathbb{D}) \longrightarrow L_{a}^{2}(\mathbb{D})
$$

has

$$
\begin{aligned}
& X_{e}=\left\{f \circ \varphi_{m} k_{m} ; f \in L_{a}^{2}(\mathbb{D}), f(-z)=f(z)\right\}, \\
& X_{o}=\left\{f \circ \varphi_{m} k_{m} ; f \in L_{a}^{2}(\mathbb{D}), f(-z)=-f(z)\right\},
\end{aligned}
$$

as its only two proper reducing subspaces, where $\varphi_{m}=(m-$ $z) /(1-\bar{m} z)$.
Theorem 7. The restrictions of $T_{\varphi}$ on $X_{e}$ and $X_{o}$ are unitarily equivalent to $T_{\psi(\sqrt{w})}$ on $L_{a}^{2}(G,(1 / 4|w|) d A(w))$ and $L_{a}^{2}(G,(1 / 4) d A(w))$, respectively, where $\psi=\varphi \circ \varphi_{m}$.

Proof. Now we define the following operators:

$$
\begin{aligned}
L_{a}^{2}\left(G, \frac{1}{4} d A(w)\right) & \longrightarrow X_{o} \\
V_{o}: f(w) & \longmapsto\left(u f\left(u^{2}\right)\right) \circ \varphi_{m}(z) k_{m}(z), \\
L_{a}^{2}\left(G, \frac{1}{4|w|} d A(w)\right) & \longrightarrow X_{e} \\
V_{e}: f(w) & \longmapsto f\left(u^{2}\right) \circ \varphi_{m}(z) k_{m}(z) .
\end{aligned}
$$

Then $V_{o}, V_{e}$ are two isometries on $X_{o}, X_{e}$, respectively. As a fact, by changing of variables two times, $\left(u=\varphi_{m}(z)\right.$, $\varphi_{m}^{\prime}(z)=\left(1-|m|^{2}\right) /(1-\bar{m} z)^{2}=k_{m}(z)$ for the first time; $z^{2}=w,|d z / d w|=|1 / 2 \sqrt{w}|$ for the second time), we have

$$
\begin{aligned}
\left\|V_{o} f(w)\right\|^{2} & =\left\|\left(u f\left(u^{2}\right)\right) \circ \varphi_{m}(z) k_{m}(z)\right\|^{2} \\
& =\int_{\mathbb{D}}\left|\left(u f\left(u^{2}\right)\right) \circ \varphi_{m}(z) k_{m}(z)\right|^{2} d A(z) \\
& =\int_{\mathbb{D}}\left|u f\left(u^{2}\right)\right|^{2} d A(u) \\
& =\int_{\mathbb{D}}|\sqrt{w} f(w)|^{2}\left|\frac{d z}{d w}\right|^{2} d A(w) \\
& =\int_{\mathbb{D}}|\sqrt{w} f(w)|^{2}\left|\frac{1}{2 \sqrt{w}}\right|^{2} d A(w) \\
& =\int_{\mathbb{D}}|f(w)|^{2} \frac{1}{4} d A(w)=\|f(w)\|^{2},
\end{aligned}
$$

so $V_{o}$ is a isometry. And

$$
\begin{aligned}
\left\|V_{e} f(w)\right\| & =\left\|\left(f\left(u^{2}\right)\right) \circ \varphi_{m}(z) k_{m}(z)\right\|^{2} \\
& =\int_{\mathbb{D}}\left|\left(f\left(u^{2}\right)\right) \circ \varphi_{m}(z) k_{m}(z)\right|^{2} d A(z) \\
& =\int_{\mathbb{D}}\left|f\left(u^{2}\right)\right|^{2} d A(u) \\
& =\int_{\mathbb{D}}|f(w)|^{2}\left|\frac{d z}{d w}\right|^{2} d A(w) \\
& =\int_{\mathbb{D}}|f(w)|^{2}\left|\frac{1}{2 \sqrt{w}}\right|^{2} d A(w) \\
& =\int_{\mathbb{D}}|f(w)|^{2} \frac{1}{4|w|} d A(w)=\|f(w)\|^{2} ;
\end{aligned}
$$

it shows that $V_{e}$ is a isometry. Moreover, for any $f(w) \epsilon$ $L_{a}^{2}(G,(1 / 4|w|) d A(w))$, we have

$$
\begin{aligned}
T_{\varphi} V_{e} f(w) & =\varphi(z) f\left(\varphi_{m}^{2}(z)\right) k_{m}(z) \\
& =\psi\left(\varphi_{m}(z)\right) f\left(\varphi_{m}^{2}(z)\right) k_{m}(z),
\end{aligned}
$$


where $\psi(z)=\varphi\left(\varphi_{m}(z)\right)$ and $m$ is the midpoint between $a$ and $b$. On the other hand,

$$
\begin{aligned}
V_{e} T_{\psi(\sqrt{w})} f(w) & =V_{e}(\psi(\sqrt{w}) f(w)) \\
& =\psi\left(\varphi_{m}(z)\right) f\left(\varphi_{m}^{2}(z)\right) k_{m}(z) .
\end{aligned}
$$

Note that $\psi(\sqrt{w})$ is a function in $L_{a}^{2}(G,(1 / 4|w|) d A(w))$. For $f(w) \in L_{a}^{2}(G,(1 / 4) d A(w))$, one can prove similarly that

$$
T_{\varphi} V_{\mathrm{o}} f(w)=V_{o} T_{\psi(\sqrt{w})} f(w)
$$

\subsection{Weighted Shift Models}

Proposition 8. The restrictions of $T_{z^{2}}$ on its minimal reducing subspaces are one-side weighted shifts, and they are not unitarily equivalent to each other.

Proof. We know that

$$
\begin{aligned}
& \mathscr{M}_{0}=\left\{\sum_{k=0}^{\infty} a_{k} z^{2 k} ; \sum_{k=0}^{\infty}(2 k+1)\left|a_{k}\right|^{2}<\infty\right\}, \\
& \mathscr{M}_{1}=\left\{\sum_{k=0}^{\infty} b_{k} z^{2 k+1} ; \sum_{k=0}^{\infty}(2 k+2)\left|b_{k}\right|^{2}<\infty\right\}
\end{aligned}
$$

are the two minimal reducing subspaces of $T_{z^{2}}$ on the Bergman space $L_{a}^{2}(\mathbb{D})$. It is clear that

$$
\begin{gathered}
T_{z^{2}}\left(\sqrt{2 k+1} z^{2 k}\right)=\sqrt{\frac{2 k+1}{2 k+3}}\left(\sqrt{2 k+3} z^{2(k+1)}\right), \\
T_{z^{2}}\left(\sqrt{2 k+2} z^{2 k+1}\right)=\sqrt{\frac{k+1}{k+2}}\left(\sqrt{2 k+4} z^{2(k+1)+1}\right),
\end{gathered}
$$

so $\left.T_{z^{2}}\right|_{\mathscr{M}_{0}}$ and $\left.T_{z^{2}}\right|_{\mathscr{M}_{1}}$ are weighted shifts with the weight sequences $\{\sqrt{(2 k+1) /(2 k+3)}\}$ and $\{\sqrt{(k+1) /(k+2)}$, respectively. It is clear that $\left.T_{z^{2}}\right|_{\mathscr{M}_{0}}$ is not unitarily equivalent to $\left.T_{z^{2}}\right|_{\mathscr{M}_{1}}$ by Lemma 1 .

Proposition 9. The restrictions of $T_{z^{2}}$ on its minimal reducing subspaces are one-side weighted shifts, and they are similar to each other.

Proof. Let $\mathscr{M}_{0}$ and $\mathscr{M}_{0}$ be the minimal reducing subspaces of $T_{\varphi}$; we know that $\left.T_{\varphi}\right|_{\mathscr{M}_{1}}$ is the weighted shift with weight $\{\sqrt{(2 k+1) /(2 k+3)}\}_{k=0}^{\infty}$ and $\left.T_{\varphi}\right|_{\mathscr{M}_{1}}$ is the weighted shift with weight $\{\sqrt{(k+1) /(k+2)}\}_{k=0}^{\infty}$. By a direct computation, we have

$$
\begin{aligned}
& \prod_{k=0}^{n} \frac{\sqrt{(2 k+1) /(2 k+3)}}{\sqrt{(k+1) /(k+2)}}=\frac{\prod_{k=0}^{n} \sqrt{(2 k+1) /(2 k+3)}}{\prod_{k=0}^{n} \sqrt{(k+1) /(k+2)}} \\
& \quad=\sqrt{\frac{n+2}{2 n+3}} .
\end{aligned}
$$

So for any $n$, we have

$$
\sqrt{\frac{1}{2}} \leq \prod_{k=0}^{n} \frac{\sqrt{(2 k+1) /(2 k+3)}}{\sqrt{(k+1) /(k+2)}} \leq \sqrt{\frac{2}{3}}
$$

since $\sqrt{(n+2) /(2 n+3)}$ is decreasing as $n$ increases to infinity. It implies that $\left.T_{\varphi}\right|_{\mathscr{M}_{0}}$ is similar to $\left.T_{\varphi}\right|_{\mathscr{M}_{1}}$ by Lemma 2 .

Remark 10. $\left.T_{\varphi}\right|_{\mathscr{M}_{1}}$ is just the Bergman shift, so we know that $T_{\varphi}$ is similar to the direct sum of two copies of the Bergman shift.

3.4. For Reducing Subspace of $M_{z^{n}}$

Theorem 11. The slit disc models for restrictions of $T_{z^{n}}$ are the $T_{w}$ on the following $n$ spaces:

$$
L_{a}^{2}\left(G, \frac{1}{n^{2}|w|^{2(n-k-1) / n}} d A(w)\right),
$$

$$
k=0,1, \ldots, n-1 \text {. }
$$

Proof. Let

$$
\mathscr{M}_{k}^{n}=\bigvee\left\{z^{m} ; m \equiv k \bmod n\right\}, \quad k=0,1, \ldots, n-1 .
$$

We define the following $n$ operators, for $k=0,1, \ldots, n-1$ :

$$
\begin{aligned}
L_{a}^{2}\left(G, \frac{1}{n^{2}|w|^{2(n-k-1) / n}} d A(w)\right) & \longrightarrow \mathscr{M}_{k}^{n} \\
V_{k}^{n}: f(w) & \longmapsto z^{k} f\left(z^{n}\right),
\end{aligned}
$$

and then we can show that $V_{k}^{n}$ are isometries; as a fact, by changing of variable $w=z^{n},|d z / d w|=\left|1 / n \sqrt[n]{w^{n-1}}\right|$, so

$$
\begin{aligned}
\left\|V_{k}^{n} f(w)\right\|^{2} & =\left\|z^{k} f\left(z^{n}\right)\right\|^{2}=\int_{\mathbb{D}}\left|z^{k} f\left(z^{n}\right)\right|^{2} d A(z) \\
& =\int_{\mathbb{D}}\left|w^{k / n} f(w)\right|^{2}\left|\frac{d z}{d w}\right|^{2} d A(w) \\
& =\int_{\mathbb{D}}|w|^{2 k / n}|f(w)|^{2}\left|\frac{1}{n \sqrt[n]{w^{n-1}}}\right|^{2} d A(w) \\
& =\int_{\mathbb{D}}|f(w)|^{2} \frac{1}{n^{2}|w|^{2(n-k-1) / n}} d A(w) \\
& =\|f(w)\|^{2} .
\end{aligned}
$$

Moreover, for any $f(w) \in L_{a}^{2}\left(G,\left(1 / n^{2}|w|^{2(n-k-1) / n}\right) d A(w)\right)$, we have

$$
\begin{aligned}
T_{z^{n}} V_{k}^{n} f(w) & =T_{z^{n}}\left(z^{k} f\left(z^{n}\right)\right)=z^{n+k} f\left(z^{n}\right) \\
& =z^{k}\left(z^{n} f\left(z^{n}\right)\right)=V_{k}^{n} T_{w} f(w) .
\end{aligned}
$$

Remark 12. $r^{\alpha} d A(z)$ is a finite measure on $G$ for $\alpha>-2$, so $\left(1 / n^{2}|w|^{2(n-k-1) / n}\right) d A(w)$ is a finite measure on $G$. 
Proposition 13. The restrictions of $T_{z^{n}}$ on its minimal reducing subspaces are one-side weighted shifts, and they are not unitarily equivalent to each other.

Proof. For the Bergman space $L_{a}^{2}(G,(1)$ $\left.\left.n^{2}|w|^{2(n-k-1) / n}\right) d A(w)\right)$ and any $j \geq 0$, we have

$$
\begin{aligned}
\left\|w^{j}\right\|^{2} & =\int_{G}\left|w^{j}\right|^{2} \frac{1}{n^{2}|w|^{2(n-k-1) / n}} d A(w) \\
& =\frac{1}{n^{2} \pi} \int_{0}^{2 \pi} d \theta \int_{0}^{1} r^{2 j+1-2(n-k-1) / n} d r \\
& =\frac{1}{n(n j+k+1)},
\end{aligned}
$$

that is, $\|w\|=\sqrt{1 / n(n j+k+1)}$. It implies that

$$
\begin{aligned}
& T_{w}\left(\sqrt{n(n j+k+1)} w^{j}\right) \\
& =\sqrt{\frac{n j+k+1}{n(j+1)+k+1}}\left(\sqrt{n[n(j+1)+k+1]} w^{j+1}\right),
\end{aligned}
$$

that is, $T_{w}$, and so $\left.T_{\varphi}\right|_{M_{k}^{n}}$ is the weighted shift with weight $\{\sqrt{(n j+k+1) /(n(j+1)+k+1)}\}$, and so $T_{w}$ is bounded and $\left.T_{\varphi}\right|_{M_{k}^{n}}$ is not unitarily equivalent to $\left.T_{\varphi}\right|_{M_{j}^{n}}$ when $k \neq j$.

Proposition 14. The restrictions of $T_{z^{n}}$ on its minimal reducing subspaces are one-side weighted shifts, and they are similar to each other.

Proof. $\left.T_{\varphi}\right|_{M_{k}^{n}}$ and $\left.T_{\varphi}\right|_{M_{\ell}^{n}}$ are the one-side weighted shift with weights $\{\sqrt{(n j+k+1) /(n(j+1)+k+1)}\}$ and $\{\sqrt{(n j+\ell+1) /(n(j+1)+\ell+1)}\}$, where $0 \leq k<\ell \leq n-1$. So, for any $m$,

$$
\begin{aligned}
& \prod_{j=0}^{m} \frac{\sqrt{(n j+k+1) /(n(j+1)+k+1)}}{\sqrt{(n j+\ell+1) /(n(j+1)+\ell+1)}} \\
& =\frac{\prod_{j=0}^{m} \sqrt{(n j+k+1) /(n(j+1)+k+1)}}{\prod_{j=0}^{m} \sqrt{\frac{n j+\ell+1}{n(j+1)+\ell+1}}} \\
& =\sqrt{\frac{k+1}{\ell+1} \sqrt{\frac{n(m+1)+\ell+1}{n(m+1)+k+1}} .}
\end{aligned}
$$

Then,

$$
\begin{aligned}
0 & <\sqrt{\frac{k+1}{\ell+1}} \leq \prod_{j=0}^{m} \frac{\sqrt{(n j+k+1) /(n(j+1)+k+1)}}{\sqrt{(n j+\ell+1) /(n(j+1)+\ell+1)}} \\
& \leq \sqrt{\frac{k+1}{\ell+1}} \sqrt{\frac{n+\ell+1}{n+k+1}}
\end{aligned}
$$

since $(n(m+1)+\ell+1) /(n(m+1)+k+1)$ is decreasing as $m$ increases to infinity. It implies that $\left.T_{z^{n}}\right|_{M_{k}^{n}}$ is similar to $\left.T_{z^{n}}\right|_{M_{\ell}^{n}}$ for any $k \neq \ell$.

Remark 15. For any $0 \leq \ell \leq n-1,\left.T_{z^{n}}\right|_{M_{\ell}^{n}}$ is similar to $\left.T_{z^{n}}\right|_{M_{0}^{n}}$ which is the Bergman shift, and so $T_{z^{n}}$ is similar to the direct sum of $n$ copies of the Bergman shift.

\section{Conflicts of Interest}

The authors declare that there are no conflicts of interest regarding the publication of this paper.

\section{Acknowledgments}

The authors thank R. G. Douglas and D. K. Keshari for the helpful discussions. Anjian $\mathrm{Xu}$ is supported in part by Scientific and Technological Research Program of Chongqing Municipal Education Commission (Grant no. KJ1500938), Chongqing Science and Technology Commission (Grant no. CSTC2015jcyjA00045), and NSF of China (11501068). Yang Zou is supported in part by Scientific and Technological Research Program of Chongqing Municipal Education Commission (Grant no. KJ1501414).

\section{References}

[1] J. Thomson, "The commutant of a class of analytic Toeplitz operators. II," Indiana University Mathematics Journal, vol. 25, no. 8, pp. 793-800, 1976.

[2] J. E. Thomson, "The commutant of a class of analytic Toeplitz operators," American Journal of Mathematics, vol. 99, no. 3, pp. 522-529, 1977.

[3] K. Zhu, "Reducing subspaces for a class of multiplication operators," Journal of the London Mathematical Society. Second Series, vol. 62, no. 2, pp. 553-568, 2000.

[4] K. Guo, S. Sun, D. Zheng, and C. Zhong, "Multiplication operators on the Bergman space via the Hardy space of the bidisk," Journal Fur Die Reine Und Angewandte Mathematik, vol. 628, pp. 129-168, 2009.

[5] R. G. Douglas, S. Sun, and D. Zheng, "Multiplication operators on the Bergman space via analytic continuation," Advances in Mathematics, vol. 226, no. 1, pp. 541-583, 2011.

[6] R. G. Douglas, M. Putinar, and K. Wang, "Reducing subspaces for analytic multipliers of the Bergman space," Journal of Functional Analysis, vol. 263, no. 6, pp. 1744-1765, 2012.

[7] G. Cassier and I. Chalendar, "The group of the invariants of a finite Blaschke product," Complex Variables. Theory and Application. An International Journal, vol. 42, no. 3, pp. 193-206, 2000.

[8] A. Tikaradze, "Multiplication operators on the Bergman spaces of pseudoconvex domains," New York Journal of Mathematics, vol. 21, pp. 1327-1345, 2015.

[9] R. G. Douglas and Y.-S. Kim, "Reducing subspaces on the annulus," Integral Equations and Operator Theory, vol. 70, no. 1, pp. 1-15, 2011.

[10] S. D. Fisher, Function Theory on Planar Domains, A WileyInterscience Publication, John Wiley \& Sons, Inc., New York, NY, USA, 1983. 
[11] R. G. Douglas, D. K. Keshari, and A. Xu, "Generalized bundle shift with application to multiplication operator on the Bergman space," Journal of Operator Theory, vol. 75, no. 1, pp. 3-19, 2016.

[12] M. B. Abrahamse and R. G. Douglas, "A class of subnormal operators related to multiply-connected domains," Advances in Mathematics, vol. 19, no. 1, pp. 106-148, 1976.

[13] R. G. Douglas, "Operator theory and complex geometry," Extracta Mathematicae, vol. 24, no. 2, pp. 135-165, 2009.

[14] J. Hu, S. Sun, X. Xu, and D. Yu, "Reducing subspace of analytic Toeplitz operators on the Bergman space," Integral Equations and Operator Theory, vol. 49, no. 3, pp. 387-395, 2004.

[15] R. Kelley, Weighted Shifts on Hilbert Space [Thesis], University of Michigan, Ann Arbor, Mich, USA, 1966.

[16] W. T. Ross, "Invariant subspaces of Bergman spaces on slit domains," The Bulletin of the London Mathematical Society, vol. 26, no. 5, pp. 472-482, 1994.

[17] A. Aleman, N. S. Feldman, and W. T. Ross, The Hardy Space of a Slit Domain, Frontiers in Mathematics, Birkhauser Basel, 2009. 


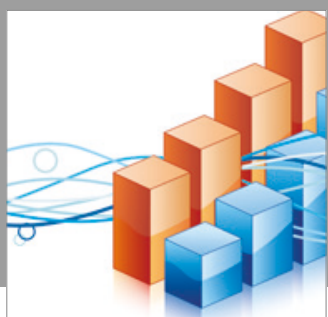

Advances in

Operations Research

vatersals

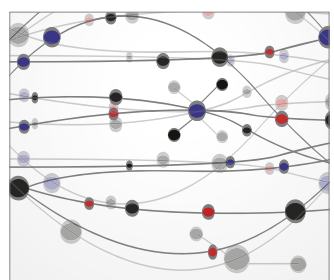

\section{The Scientific} World Journal
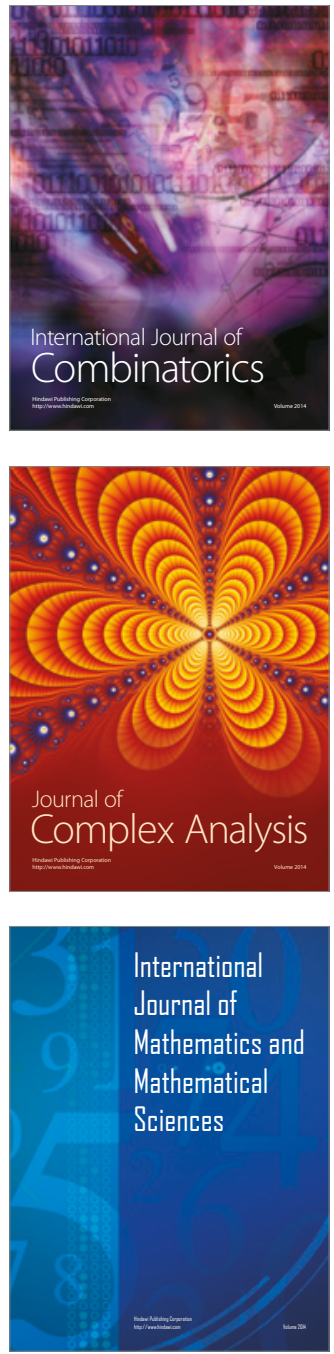
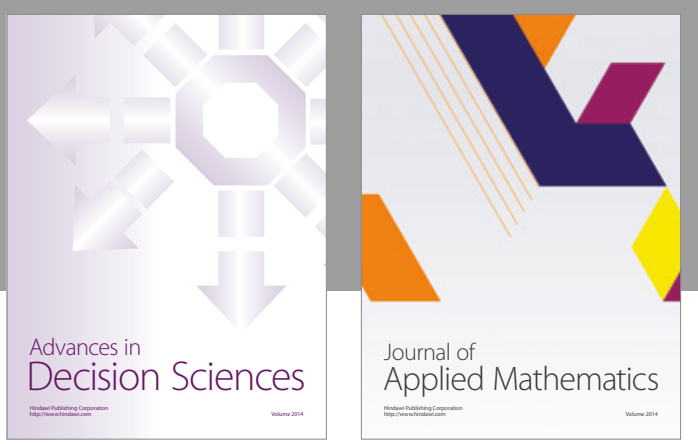

Algebra

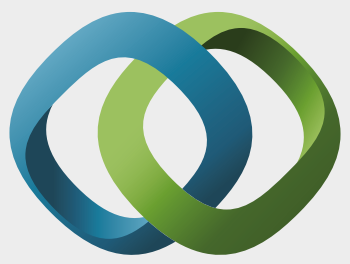

\section{Hindawi}

Submit your manuscripts at

https://www.hindawi.com
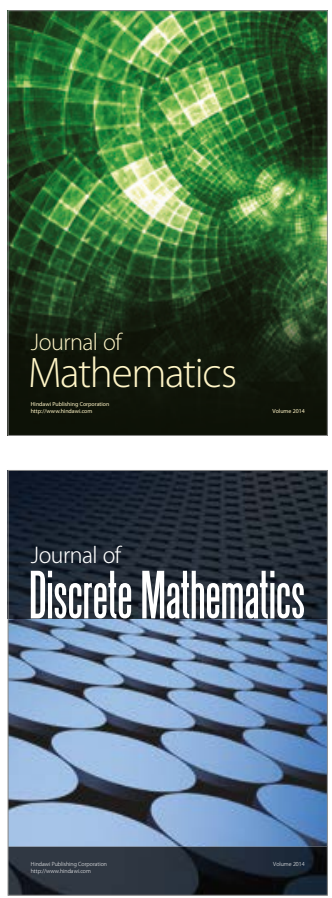

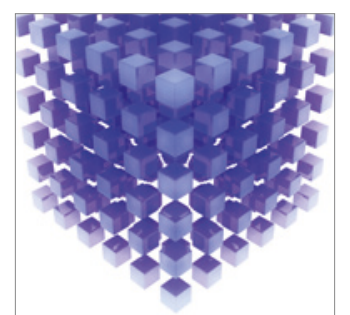

Mathematical Problems in Engineering
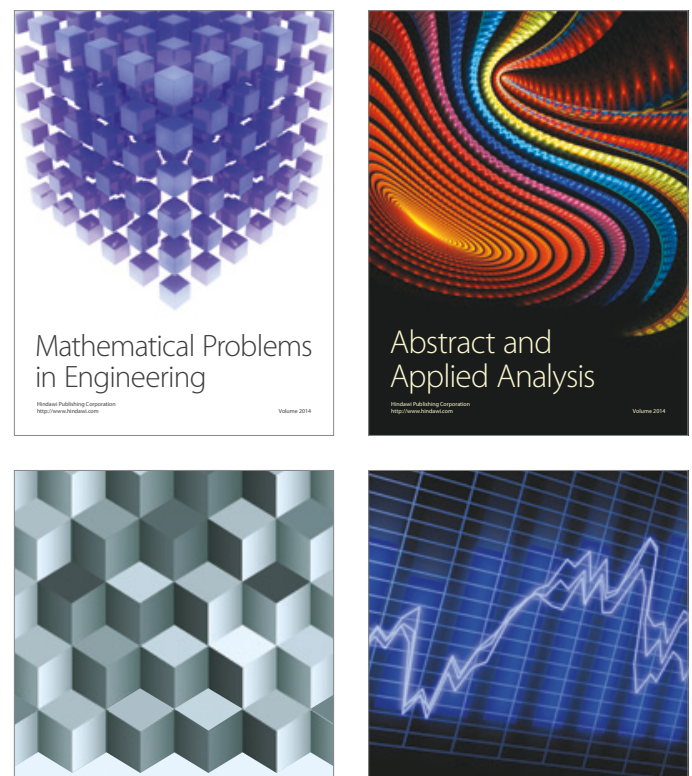

Journal of

Function Spaces

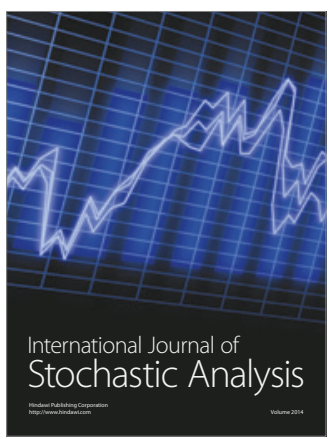

Probability and Statistics
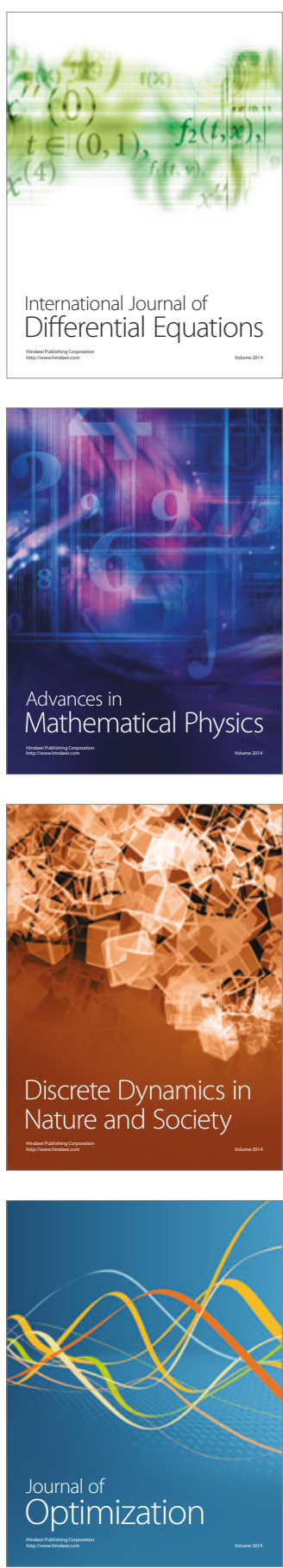